\title{
A DYNAMIC DISPERSION INSERT IN THE FERMILAB MAIN INJECTOR FOR MOMENTUM COLLIMATION*
}

\author{
D.E. Johnson", FNAL, Batavia, IL, USA
}

\begin{abstract}
The Fermilab Main Injector (MI) accelerator is designed as a FODO lattice with zero dispersion straight sections. A scheme will be presented that can dynamically alter the dispersion of one of the long straight sections to create a non-zero dispersion straight section suitable for momentum collimation. During the process of slip stacking DC beam is generated which is lost during the first few milliseconds of the ramp. A stationary massive primary collimator/absorber with optional secondary masks could be utilized to isolate beam loss due to uncaptured beam.
\end{abstract}

\section{MAIN INJECTOR LATTICE}

\section{Ring Lattice}

The MI is $3.3 \mathrm{~km}$ in circumference and was designed as a pure FODO lattice with eight dispersion free straight sections of either 3, 4, or 8 half-cells in length. The halfcell length, $L_{a}$, in the arcs and straight sections are 17.288 $\mathrm{m}$. The operational tunes $(\mathrm{H} / \mathrm{V})$ are 26.425 and 25.415. The arc dipoles are 240 inches with a peak excitation on $1.72 \mathrm{~T}$ and the arc and straight section quads are 84 inches (IQB) with a peak gradient of $19.6 \mathrm{~T} / \mathrm{m}$. The lattice

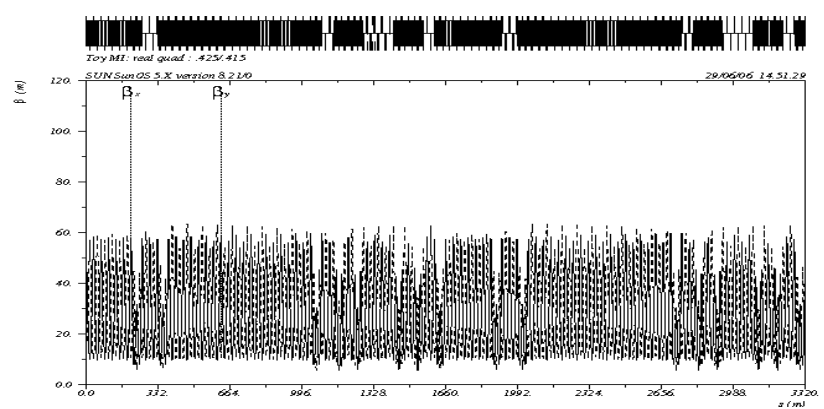

Figure 1. Lattice functions of the current MI

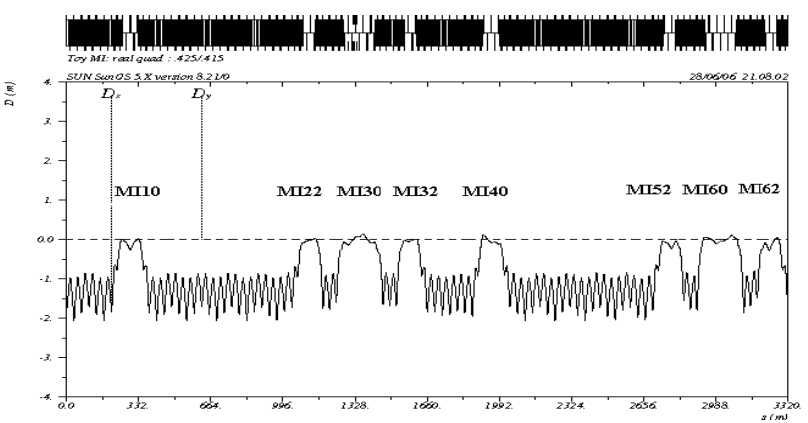

Figure 2: Dispersion function of the current MI.

* Operated by Fermi Research Alliance, LLC under contract No. DEAC02-07CH11359 with the United States Department of Energy \#dej@fnal.gov functions and dispersion of the current ring are shown in Figures 1 and 2.

\section{Straight Section and Dispersion Suppression}

Each straight section is bordered by a four half-cell insert called a "dispersion suppressor". The straight sections are "90 degree FODO cells" with similar lengths and lattice functions as the arc cells. The insert matches the lattice functions between the arc and the straight section and suppresses the dispersion generated in the arc dipoles. The technique used to suppress the dispersion utilized a variation of the "half-dipole strength scheme"[1]. For geometry reasons the bending in the dispersion suppressors, $\theta^{\prime}$, was reduced by $2 / 3$, so to maintain the dispersion suppression, the half-cell length in the dispersion suppressor, $\mathrm{L}_{\mathrm{d}}$, was reduced by $3 / 4$ such that the product $L_{d} \theta^{\prime}=1 / 2 L_{a} \theta$. To keep the dispersion suppressor matched to the arc and straight section, the integrated gradients of the quads within the dispersion suppressor were increased to 116 inches (IQD) and those at the arc (or straight section) interface were increased to 100 inches (IQC).

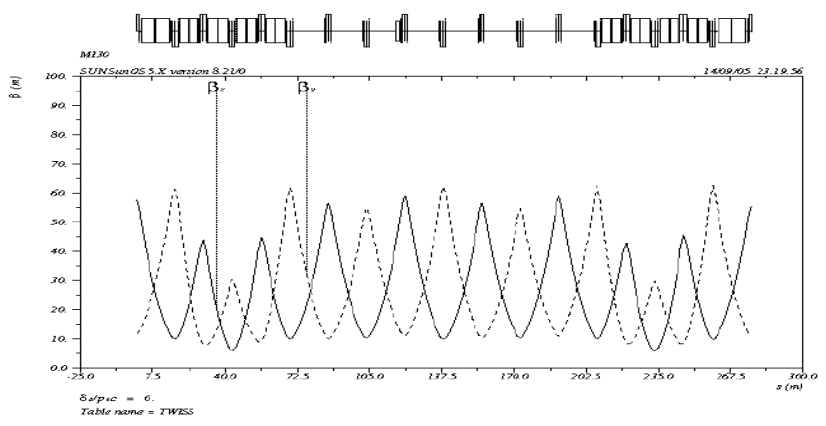

Figure 3: Current MI30 straight section lattice.

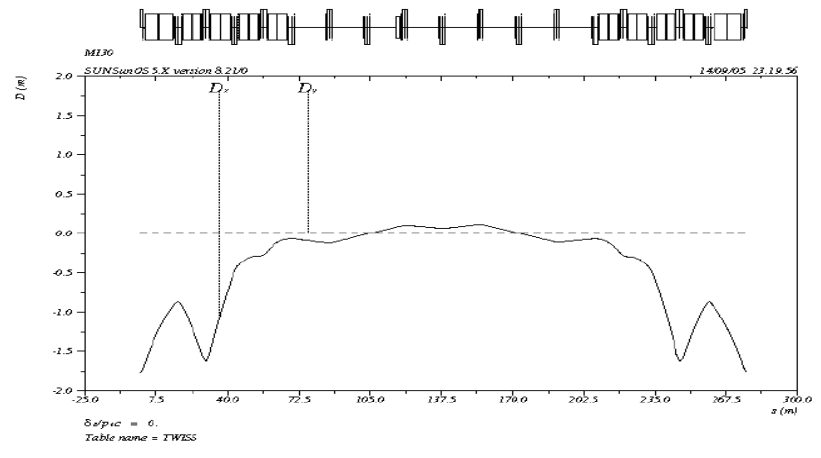

Figure 4: Current MI30 straight section dispersion.

Since all quads are on a single focusing or defocusing bus, a 16 turn trim coil was installed in the IQC and IQD 
quads to keep the IQC(D)/IQB ratio constant at all energies. To date, these trim coils have not been powered.

\section{UN-SUPPRESSING THE DISPERSION}

The proposal is to utilize the trim coils in the IQC and IQD magnets to "un-suppress" the dispersion such that a local symmetric dispersion wave within the MI30 straight section is created. This local dispersion bump can be programmed in time such that it is used only when needed (i.e. on event). The phase of this dispersion wave is such that a location within MI30 becomes the limiting momentum aperture (with the aid of a closed orbit bump, if needed) for the slip-stacking cycle or any cycle that we wish to provide momentum collimation. The magnitude of the dispersion wave produces a value of dispersion up to $15 \%$ greater than the maximum disperaion in the arcs.

The procedure is to power the trim coils on either side of the insertion symmetrically to produce a "local" symmetrical insert. This yields five "quad circuits" using the trim coils in the IQC and IQD quads. In addition, trim quads, utilized for half integer extraction are inatalled at each of the 7 IQB quads in the straight section (Q302Q308) and powered in 4 circuits symmetrically around the center of the straight section. This yields a total of 9 circuits. In addition, the trim coils in the dispersion suppressor quads, IQC's and IQD's, are connected in four loops, focussing and defocussing for each quad type. These will be used to smooth the lattice functions upon retuning the machine tune.The lattice code MAD is then used to adjust these circuits to produce the desired insert matched to the ring.

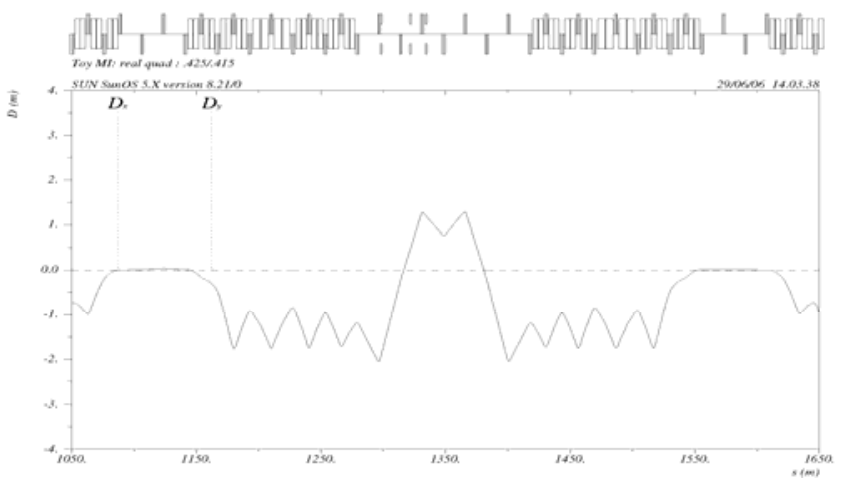

Figure 5: Resultant MI30 dispersion after un-suppressing and re-tuning lattice.

The desired dispersion (at Q302 and Q308), the maximum beta in region, and matching constraints at the the end of the insert are specified and then using the trim coils around the insert and the trim quads in the straight section, the required gradients are found. This changes the phase advance within the straight section an hince the total machine tune. The main quads and trim coils in the IQC's and IQD's in the remainder of the ring are adjusted to compensate phase shift and minimize lattice distortion around the ring. The main QF and QD bus may be used to re-adjust tune to the desired value. The required currents in the trim quads $(<10 \mathrm{~A})$ and trim coils $(<15 \mathrm{~A})$ are well with in their range.

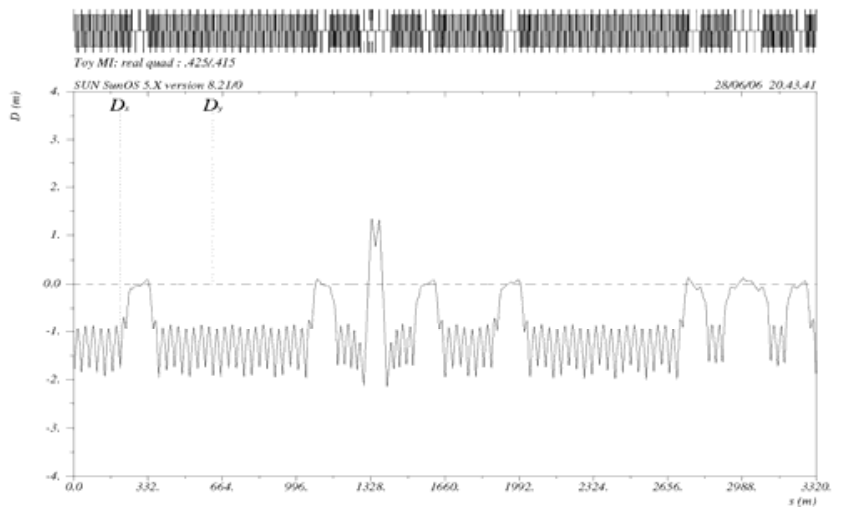

Figure 6: Ring disperaion after creating $2.2 \mathrm{~m}$ dispersion in MI30.

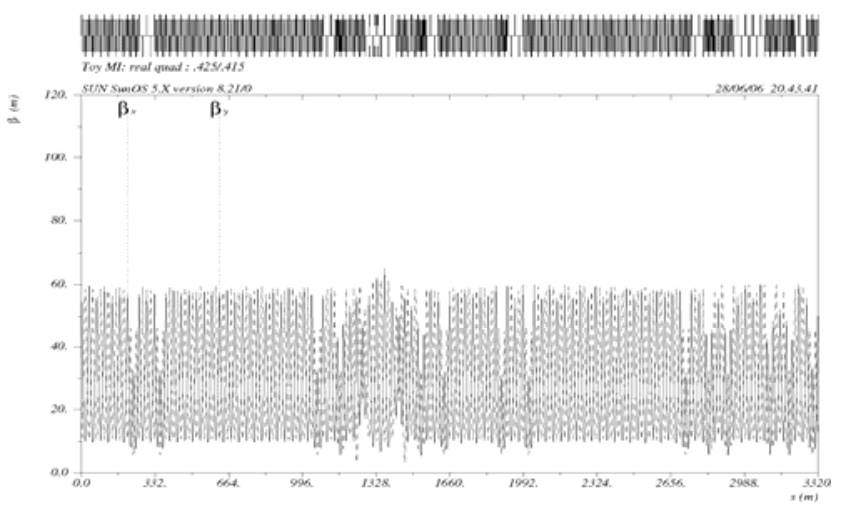

Figure 7: Ring lattice functions after retuning to nominal tune.

Figures 6 and 7 show the ring dispersion and lattice functions for the solution depicted in figure 5 .

\section{MOMENTUM COLLIMATION}

\section{Uncaptured beam}

The horizontal motion of a particle in a dispersive region with a dispersion, $D(s)$, is given by

$$
\begin{aligned}
x(s, t)=\sqrt{\frac{\varepsilon \beta(s)}{\gamma \beta(t)}} \cos \phi(t)+D(s) \delta & +\langle x(s, t)\rangle_{c o} \\
& \left.+D(s) \frac{d p}{p}(t)\right\}
\end{aligned}
$$

The first two terms describe the distribution of particles, whether captured or un-captured, due to the transverse emittance, $\varepsilon$, and machine lattice function, $\beta(s)$, and due to the momentum distribution, $\delta$, at a location with dispersion $\mathrm{D}(\mathrm{s})$. The $\cos \phi(\mathrm{t})$ term is just the relative phase of the particle due to the fractional tune. The third term, $<\mathrm{x}(\mathrm{s}, \mathrm{t})>\mathrm{co}$, is the horizontal position of the closed orbit at location $\mathrm{s}$ and time. The last term only pertains to an un-captured particle and it is the orbit deviation of this un-captured particle relative to the central momentum of a captured particle at the location $\mathrm{s}$ with a dispersion $\mathrm{D}(\mathrm{s})$ at 
time $\mathrm{t}$ during the acceleration cycle. This difference in momentum between the un-captured beam and the change in momentum of the captured beam due to the ramp is given by

$$
\frac{d p}{p}(t)=\frac{p_{u}-p_{c}(t)}{p_{c}(t)}
$$

where $p_{u}$ is the momentum of the un-captured particle and $p_{c}$ is the momentum of the synchronous particle during the ramp. The time dependence of this momentum deviation is determined by the MI ramp structure. In our case, the term, $\frac{d p}{p}(t)$, will always be negative relative to the momentum of the captured particles on the ramp. Figure 8 shows the momentum and $d p / p$ for the first 200 ms of a typical MI ramp. The time into the ramp where beam loss is measured are indicated.

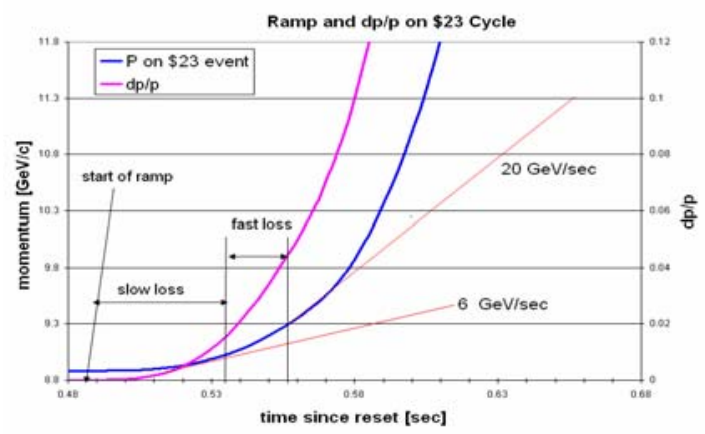

Figure 8 . Momentum and $\mathrm{dp} / \mathrm{p}$ at the start of a typical MI ramp. Straight lines are only for reference.

Figure 9 shows the rate and displacement of the uncaptured beam during the first part of the MI ramp depicted in figure 8 . Here it is seen that at $.53 \mathrm{sec}$ when the fast loss is observed, the uncaptured particle has an offset of of about $20 \mathrm{~mm}$ and is moving toward the inside of the ring at $20 \mathrm{micron} /$ turn with a transverse velocity of $2 \mathrm{~m} / \mathrm{sec}$.

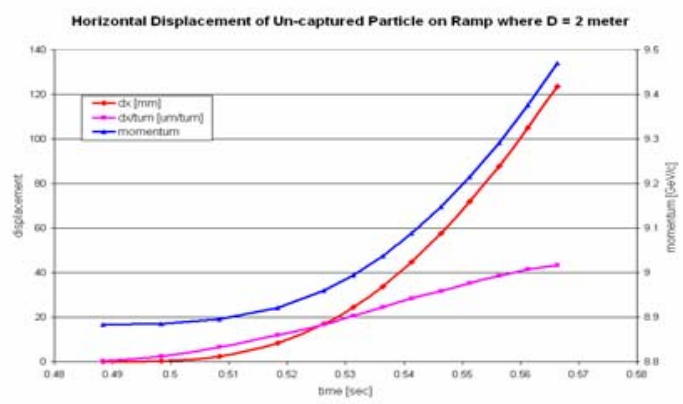

Figure 9. Displacement of uncaptured beam at the start of the MI ramp due only to the momentum difference between captured and un-captured particle at a dispersion of two meters.

\section{Imapct Parameter}

The efficiency of a collimation system is dependent particle energy, collimator length and material, and on how far from the edge of a primary collimator (in a single stage system ) or the secondary collimator (of a two-stage system) a particle is intercepted, i.e. the impact parameter.
If the impact parameter is too small, the particle may be scattered out of the collimator to be potentially lost elsewhere. Halo transverse diffusion velocities in Colliders are typically in the range of $1.5 \mathrm{micron} / \mathrm{sec}$ [2] which is on the order of $10^{6}$ smaller than due to motion of an un-captured particle. The small transverse diffusion velocity in Colliders give rise to impact parameters of .1 to .15 micron. In the Tevatron, the impact parameter grows linearly with transverse difusion velocity.[2]

\section{Utility for Collimation}

The creation of non-zero dispersion in a long straight section could lead to increased collimation efficieny and momentum selectiveness for either a two stage collimation scheme[2] or a single stage collimation scheme.

For a two-stage system, a non-zero dispersion at the secondary collimators will allow the collimator jaw to be farther from the circulating beam, thus reducing the amount of unattended transverse collimation. This also reduces the sensitivity to injection oscillations and orbit excursions during the initial stages of acceleration.[3]

The large transverse velocity of the uncaptured beam implies larger impact parameters which could be utilized in a single stage collimation scheme (without a thin primary scraper). Here, a long, stationary, heavy metal jaw and absorber, with the required shielding is utitlized at the peak dispersion. The collimator jaw would be parallel to the closed orbit and offset sufficiently that the collimator did not intefer with circulating beam, but would become the limitimg momentum aperture. If necessary a closed orbit bump could be uitlized to either move the uncaptured beam into the collimator or steer the circulating beam away from the collimator. The collimator would be fixed, but alignable. The ends of the collimator would be tapered to increase inpact parameter and spread energy deposition. Utilization of "masks" and shield walls would be considered if needed to minimize activation of accelerator components.

\section{SUMMARY}

A sheme of creating dispersion in the MI long straight section MI30[4] has been presented and its utility in either a single or two stage collimation system has been outlined. Additional simulations are necessary to fully validate the process.

\section{REFERENCES}

[1] R. Brinkmann, "Insertions", Proceedings of Cern Acceleratoe School $2^{\text {nd }}$ General Accelerator Physics Course, Aarhus, Denmark, Sept. 1968, p. 52.

[2] N.M. Mokhov, "Beam Collimation at Hadron Colliders", FERMILAB-Conf-03/220 July 2003.

[3] A.I. Drozhdin, et. al., "Collimation System Design for the Beam Loss Localization with Slipstacking Injection in the Fermilab Main Injector, TUPAS016 this conference. 
[4] D.E. Johnson, "Dynamic Dispersion for MI Collimation Review", FNAL Beams-doc-2135. 